\title{
Diagnosis and Neurosurgical Management of Cerebral Nocardiosis
}

\author{
Carley Karam ${ }^{1} \quad$ Abdolreza Siadati $^{1}$ \\ ${ }^{1}$ Department of Surgery, Neurosurgery Clinical Clerkship, University \\ of North Texas Health Science Center, Medical City Hospital, \\ Fort Worth, Texas, United States \\ Address for correspondence Carley Karam, MS, Neurosurgery Clinical \\ Clerkship, University of North Texas Health Science Center, Medical \\ City Hospital, Fort Worth, TX 75204, United States \\ (e-mail: carkar@utexas.edu).
}

J Neurol Surg Rep 2021;82:e21-e24.

\begin{abstract}
Keywords

- Nocardia

- abscess

- craniotomy

- excision

- infection

- complete

- surgical

- tuberculosis

Primary Nocardia infections are uncommon gram-positive bacterial infections caused by aerobic actinomycetes and are typically regarded as opportunistic infections (only one-third of infected patients are immunocompetent). Risk factors include: glucocorticoid therapy, malignancy, organ transplant recipients, human immunodeficiency virus infections, tumor necrosis factor- $\alpha$ inhibitor therapy, diabetes mellitus, alcoholism, inflammatory bowel disease, chronic obstructive pulmonary disease, chronic granulomatous disease, and tuberculosis. The organism has a predisposition to disseminate to the central nervous system and can relapse or progress despite appropriate therapy. Treatment ranges from oral antibiotic management to multiple intravenous antibiotic therapy, with surgical intervention required for severe cases. The surgical options include aspiration or complete excision of abscess contents and capsule. In the present case, we describe the use of bilateral craniotomy with assisted image guidance and multiple abscess excision in an immunocompetent patient with systemic nocardiosis.
\end{abstract}

\section{Introduction}

Primary Nocardia infections are uncommon gram-positive bacterial infections caused by aerobic actinomycetes and are typically regarded as opportunistic infections (only one-third of infected patients are immunocompetent). ${ }^{1}$ Risk factors include: glucocorticoid therapy, malignancy, organ transplant recipients, human immunodeficiency virus infections, tumor necrosis factor- $\alpha$ inhibitor therapy, diabetes mellitus, alcoholism, inflammatory bowel disease, chronic obstructive pulmonary disease, chronic granulomatous disease, and tuberculosis. The organism has a predisposition to disseminate to the central nervous system and can relapse or progress despite appropriate therapy. ${ }^{2}$ Treatment ranges from oral antibiotic management to multiple intravenous (IV) antibiotic therapy, with surgical intervention required for severe cases. The surgical options include aspiration or complete excision of abscess contents and capsule. In the present case, we describe the use of bilateral craniotomy with assisted image guidance and multiple abscess excision in an immunocompetent patient with systemic Nocardiosis.

\section{Case Presentation}

A 58-year-old well-nourished, Caucasian male reported to the hospital after 1 week of low-grade fevers, chills, headache, and recent weight loss. He reported an episode of syncope after working in his yard in the heat that occurred a few days before the onset of his symptoms. He attributed his symptoms to being overheated in his yard and only received

December 6, 2019

accepted

June 11,2020
DOI https://doi.org/

10.1055/s-0040-1722345. ISSN 2193-6358.

\footnotetext{
(C) 2021. The Author(s).

This is an open access article published by Thieme under the terms of the Creative Commons Attribution-NonDerivative-NonCommercial-License, permitting copying and reproduction so long as the original work is given appropriate credit. Contents may not be used for commercial purposes, or adapted, remixed, transformed or built upon. (https://creativecommons.org/ licenses/by-nc-nd/4.0/) Georg Thieme Verlag KG, Rüdigerstraße 14, 70469 Stuttgart, Germany
} 
presented to the hospital after noticing increasing weakness that day. His past medical history consisted of prediabetes, hypertension, and dyslipidemia, for which he took lisinopril and a statin. He denied any history of stroke, seizures, previous brain surgery, or recent trauma. He had no past history of any immunocompromising disorders or therapies and reported no recent travel outside of the country or exposure to environmental toxins. His past family history consisted of breast cancer in his mother, Alzheimer's disease, and Parkinson's disease. He never smoked, denied using any drugs not prescribed to him, and denied drinking alcohol. He works as an AC mechanic and also does a lot of woodwork. Upon examination, the patient was alert and oriented to person, place, and time, his Glasgow Coma scale was 15, cranial nerves II-XII were intact, his strength and sensation were normal in all extremities, he had no Hoffman's sign or Babinski's sign, and deep tendon reflexes were $2+$ in bilateral biceps, brachioradialis, and Achilles, but he was hyper-reflexive in the bilateral patellas. Computed tomography of the lungs during the current presentation revealed a ring-enhancing, well-circumscribed lesion in the posterior aspect of the right upper lung lobe, appearing to be attached to the posterior pleural membrane. Magnetic resonance imaging (MRI) of the head revealed two ring-enhancing, well-circumscribed lesions, one in the left frontal lobe and one involving the right temporal and occipital horn portions of the right lateral ventricle with possible ventriculitis and a mild left to right midline shift. Blood cultures were pending; however, considering the severity of the differential diagnosis of metastatic disease versus a systemic infection with multiple abscesses, the decision was made to take the patient to surgery to perform a bilateral craniotomy with plans for biopsy versus aspiration versus excision. Preoperatively, the patient was started on IV fluids, empiric vancomycin, empiric meropenem, ondansetron, and prophylactic levetiracetam. He underwent a dedicated brain MRI with stealth protocol, a sequence that has more thin cuts than the standard MRI and is specifically used for surgical planning. Intraoperative navigational software from Medtronic was interfaced with his stealth MRI and used throughout the procedure for incision planning, depth approximation, and avoiding large underlying vessels and sinuses to greater than $1 \mathrm{~mm}$ of accuracy. ${ }^{3}$

The patient was taken to the operating room, placed under general anesthesia, intubated, and appropriate lines were placed. He was placed supine with his head secured in a Mayfield head holder frame, his head was shaved with clippers, cleansed with alcohol, prepped with betadine, and draped in standard surgical fashion. A navigational probe was used to trace the patient's bony landmarks so that his anatomy could be loaded into the system and interfaced with his stealth MRI. This interface allows the neurosurgeon to place a probe on the patient that displays the actual underlying anatomy on the system monitor, including the position of the lesion of interest in relation to the location of the probe. ${ }^{4}$ A curvilinear incision was made with a no. 10 blade from the midline frontal area to the front of the left ear after using the imaging probe to determine the area of the scalp directly over the lesion. Electrocautery was then used to free up a flap of skin and dissect through the fascia. Raney clips were used on the scalp edges for hemostasis and retractors were used for better visualization. At this point, the skull was identified, and the imaging probe was used again to approximate the borders of the lesion so that appropriate bone opening could be made. A small burr hole was drilled through the skull without injuring the underlying dural membranes or brain parenchyma. After this a craniotomy was made and bone flap preserved; FloSeal was used for hemostasis and bone wax was used on the cancellous bone for the prevention of air embolus from opened bone edges. The dural membrane was then opened and retracted, and the team proceeded to the intracranial surgery. The brain here was very swollen, and we could see dilated gyri. Using stealth, the corticectomy was made right over the area where the mass was closest to the surface. A fair amount of pus was drained and sent for gram stain. The gross specimen showed sheets of neutrophils under the microscope, but the pathologist was unable to isolate a specific organism intraoperatively. Next, we were able to go around the necrotic wall and resect the abscess wall itself. After this maneuver, the brain appeared more relaxed. Hemostasis was achieved, dura was closed, and DuraGen was applied, and the bone flap was replaced using Synthes mini plates. Evicel was applied and the area was thoroughly irrigated with antibiotic irrigation. A layered closure of the scalp was performed by closing the galea, stapling the skin, and a sterile dressing was applied. The patient tolerated the procedure well, and the team then transitioned to the second operation.

The sterile field was broken, and the patient was repositioned in the Mayfield head holder frame to best visualize the right occipital area. The right occipital parietal area was shaved with clippers, prepped, and draped. A right occipital craniotomy was performed again in the same manner as before. A new target trajectory was mapped out in the same way with the stealth imaging system, and a microscope was brought into the field to progress deeper toward the mass. There was arachnoid granulation that was inflamed secondary to the infection. We continued to work and watch for any cerebrospinal fluid or bleeding into the ventricular system. Once the abscess and its wall were fully resected, a ventricular catheter was placed directly into the occipital horn. The dura was closed with suture, and DuraGen was applied. The bone flap was replaced and fixated to the skull using Synthes mini plates. Evicel was applied and the ventricular catheter was tunneled through the skin. Next, the area was thoroughly irrigated with antibiotic irrigation and the skin was closed. No complications were observed, and blood loss was minimal. The patient was subsequently transferred to the neuro intensive care unit for postoperative care and management.

Postoperative head computed tomography (CT) on the following day showed clearing of the lesions with expected postoperative fluid and gas at the resection sites. No effacement of the basilar cisterns or CT evidence of acute ischemic infarction was present. Once his cultures resulted with Nocardia, his IV antibiotics were switched to ceftriaxone, sulfamethoxazoletrimethoprim, and linezolid. The patient did well and was set 
up for outpatient neurosurgical and infectious disease followup and visual field testing. He was continued on his IV antibiotic regimen for 4 to 6 weeks through a peripherally inserted central catheter line from home, with a switch to oral therapy after that. He was also on antiseizure medication.

\section{Literature Review}

Brain abscess is a relatively uncommon entity in the United States, with an occurrence of 1,500 to 2,500 cases per year. Since the widespread availability of CT/MRI guidance and improvement of antimicrobial therapy, the diagnosis and treatment have improved significantly. Mortality from the procedure specifically has declined from 40 to $60 \%$ in the preCT era to 0 to $10 \%$ currently. However, brain abscesses continue to carry a high morbidity and mortality rate. ${ }^{6}$ Most isolated microorganisms are Streptococcus species, Staphylococcus species, and gram-negative rods. Fungal organisms are considered rare, and Actinomyces are considered extremely rare. ${ }^{6}$

Nocardia species typically appear as delicate filamentous branching rods and must be differentiated from the anaerobe Actinomyces by acid-fast staining. Nocardia stains as acid-fast due to the mycolic acid content in its cell wall, and even though it has some characteristics of fungi, such as its branching hyphal-like appearance, it is still classified as a bacterium due to its molecular make up. There are more than 80 species in the Nocardia family that can be differentiated with molecular techniques, and new species are expected to continue to emerge. ${ }^{1}$ Nocardia species are found worldwide in soil, decaying vegetable matter, aquatic environments, and on airborne dust particles. ${ }^{1}$ Inhalation is the most common route of entry and the majority of infections involve the lung. Other modes of entry can include ingestion, which involves the gastrointestinal tract, or cutaneous infection which involves the skin and soft tissue. Hematogenous dissemination is the most common mechanism of spread within the body.

The patient presented to the hospital as an otherwise healthy, well-nourished, immunocompetent middle-aged male with a progressive systemic Nocardia infection that had formed abscesses in both his lungs and brain. His initial scans were read as metastases versus abscesses and the source of his lesions was unclear. There was not a high clinical suspicion for either metastatic disease or systemic infection with multiple abscesses before imaging; thus, his presentation was relatively mild and unique. The patient was a good surgical candidate and the decision to take a surgical approach was necessary to reach a timely diagnosis and provide definitive treatment.

Open excision was selected in this case because of the multiple brain abscesses and deep ventricle involvement. An open technique may reduce the need for additional imaging, surgical treatment, and length of antibiotic therapy. ${ }^{4}$ However, surgical excision, once the mainstay of therapy for brain abscesses, has been overshadowed by the advent of stereotactic aspiration, which has become the treatment of choice in many institutions. ${ }^{6}$ The biggest drawback of aspiration is that the abscess capsule is left intact and removal of purulent material is often incomplete. ${ }^{5}$ As a result, most patients require multiple procedures to achieve resolution of the abscess. ${ }^{6}$ Mamelak et al reported that $62 \%$ of the patients in their series required additional surgery for drainage after initial aspiration. ${ }^{6}$ Considering rising health care costs, length of hospital stay, length of antibiotic therapy, and need for repeat procedures and imaging, the older approach of complete open excision still holds an important role in the treatment of this pathology. Specifically, this case report exemplifies the positive role that complete surgical excision played in the management of this patient's condition and subsequent recovery, as he was discharged to home on postoperative day 6 and did not require any further surgeries.

In the treatment of Nocardiosis, the most recommended agent is sulfamethoxazole-trimethoprim, but isolates of the species are variably resistant, thus empiric coverage with two or three agents in patients with severe infection is recommended. ${ }^{7}$ Some species are less susceptible or resistant to sulfamethoxazole-trimethoprim; however, there have been few reports of patients failing to respond. In vitro susceptibilities and animal models of disease have demonstrated activity against Nocardia with a variety of antibiotics, including amikacin, imipenem, meropenem, third generation cephalosporins, minocycline, extended spectrum fluoroquinolones, linezolid, tigecycline, and dapsone. ${ }^{7}$ Antibiotic course is usually prolonged for 3 to 6 months because of the relapsing nature of infection. Patients on immunosuppressive therapy due to transplantation should not be taken off their immunosuppressives, but the doses should be decreased as much as possible, while patients whose immunosuppressives can be discontinued should be switched to any available alternative agents. ${ }^{2}$

In conclusion, Nocardia brain abscess is a rare problem in the United States with several different treatment options. While the most common surgical approach is stereotactic aspiration, we believe open excision was more valuable for our patient considering the locations, multiple lesions, and invasion of the ventricles with a midline shift. His outcome from surgery was favorable and complete wall excision most effectively controlled his infectious process. His initial empiric antibiotic therapy and post-culture adjustment were appropriate and well-targeted based on the review of the literature. His recovery has been promising and he will continue to follow-up in the outpatient neurosurgical clinic for the duration of his therapy.

\section{Conflict of Interest \\ None.}

\section{References}

1 Spelman D, Sexton D, Mitty J. Microbiology, epidemiology, and pathogenesis of nocardiosis. In: Post TW, editor. UpToDate [Internet]. Waltham, MA: UpToDate; 2019. Accessed July 29, 2021 from: https://www.uptodate.com/contents/microbiologyepidemiology-and-pathogenesis-of-nocardiosis

2 Lederman ER, Crum NF. A case series and focused review of nocardiosis: clinical and microbiologic aspects. Medicine (Baltimore) 2004;83(05):300-313 
3 Hanft S, Bruce J, Miller J. Craniotomy technique: approach considerations, craniotomy, craniectomy, stereotactic craniotomy. New Brunswick, NJ: Medscape; 2017. Accessed July 29, 2021 from: https://emedicine.medscape.com/article/1890449-technique

4 Gadgil N, Patel AJ, Gopinath SP. Open craniotomy for brain abscess: a forgotten experiencex003F;. Surg Neurol Int 2013;4:34

5 Connolly ES, McKhann GM, Huang J, Choudri TF, Komotar RJ, Mocco J. Fundamentals of Operative Techniques in Neurosurgery. 2nd ed. 2nd ed. New York: Thieme Medical Publishers; 2010
6 Spelman D. Treatment of nocardiosis. In: Post TW, editor. UpToDate [Internet]. Waltham, MA: UpToDate; 2019. Accessed July 29, 2021 from: https://www.uptodate.com/contents/treatment-ofnocardiosis?source=search_result\&search=nocardiosis\&selected Title=2 91

7 Brown-Elliott BA, Brown JM, Conville PS, Wallace RJ. Clinical and laboratory features of the Nocardia spp. based on current molecular taxonomy. Clin Microbiol Rev. 2006 Apr; 19(02):259-282 